To appear in Philosophical Magazine Letters

Vol. 00, No. 00, Month 20XX, 1-10

\title{
ARTICLE \\ Excited Helium under High Pressures in the Bulk and in Nanobubbles
}

\author{
N. C. Pyper ${ }^{a \dagger}$, T. C. Naginey ${ }^{\mathrm{b} * \dagger}$, P. D. Nellist ${ }^{\mathrm{b}}$, and Colm T. Whelan ${ }^{\mathrm{c}}$ \\ ${ }^{a}$ University Chemical Laboratories, Lensfield Road, Cambridge, CB2 1EW, UK; \\ ${ }^{b}$ Department of Materials, Parks Road, University of Oxford, OX13 PH; \\ ${ }^{c}$ Physics Department, Old Dominion University, Norfolk, Virginia, USA
}

( $v 5.0$ released January 2015)

\begin{abstract}
We systematically investigate the effects of intense pressures on the excitation energies of helium trapped in bubbles in order to deepen our understanding of the fundamental physics of atoms in extreme conditions. The $1 s^{2} \rightarrow 1 s 2 p$ excitation energy of a confined helium atom is known to differ from that of a free atom being greater in both the bulk liquid or solid or a bubble confined in a metallic matrix state. We compare calculations for the energy shift with both laboratory experiments for bulk systems and results derived from scanning transmission electron microscope (STEM) studies of helium nanobubbles embedded in different matrices. We find excellent agreement between our calculations and the latest extensive measurements in the bulk. However we find significant discrepancies when we compare with results deduced using the "standard" approach for analyzing STEM data. Here we show the scattering matrix element determining the intensity of this excitation in a STEM experiment is significantly affected by the same environmental factors that shift the excitation energy. Consequently there is a serious theoretical inconsistency in the way the STEM results are calculated, in that the "standard" approach depends on a supposedly known $1 s^{2} \rightarrow 1 s 2 p$ scattering cross section, whereas we show here that this cross section is itself dependent on the environment. Correcting for this inconsistency does not, in itself, improve agreement.
\end{abstract}

Keywords: electronic structure; ab initio; computer modelling; EELS

\section{Motivation}

The interaction of electrons with matter provides a powerful way to characterize materials. Electron energy-loss spectroscopy (EELS) in the scanning transmission electron microscope (STEM) allows electronic excitations of materials to be explored at high spatial resolution. Both the energy and strength of an excitation can be measured [1]. In most cases, quantitative interpretation of such data requires comparison to calculations. The excitation energies and cross-section values are often calculated making the assumption of isolated atoms (see for example [2]), whereas for most materials the atoms are in a different condition to the isolated state leading to substantially different excitation energies and cross-sections. To improve the precision to which EELS data can be quantified, it is therefore important to include the environment of the atoms in the calculation.

${ }^{\dagger}$ Both authors contributed equally to this work

*Corresponding author. Email: timothy.naginey@materials.ox.ac.uk 
The study of confined atoms is known to be of both of fundamental and technological significance [3]. An important example of the latter is the formation of helium bubbles in the metallic components of either existing fission or proposed fusion nuclear reactors. In a reactor the in-service materials will experience bombardment from radiation produced by nuclear transformations. Some of these radiations result in the formation of helium atoms within the metal. Continual bombardment causes these atoms to aggregate into bubbles trapped inside the metal where they exert very high pressures. The occurrence of such high pressure bubbles weakens the structure of the metal in these components and would, at a sufficiently high pressure, cause the material to crack [4]. Knowledge of the formation of such helium bubbles, especially of the pressures they exert, is needed to ensure that reactors are still safe after several years of operation. Since it is not possible to measure the bubble pressures directly, these must be deduced from a reliably known and tested equation of state which requires as input the bubble density or equivalently the masses and volumes of the bubbles. The pressure is highly sensitive to the density $[5,6]$ so it is important to determine the density to the highest possible accuracy. Although reliable equations of state are available and information about bubble dimensions has been derived by (S)TEM experiments, it is not possible to measure directly either the mass or the density of helium gas in the bubbles.

It is known that the $1 s^{2}$ to $1 s 2 p$ helium atom excitation energy depends on its environmant, this being revealed through STEM experiments. We show here that such dependency not only extends to the scattering cross-section but also that the latter is very significantly more environmentally sensitive than the excitation energy. Consequently it should be expected that there are significant errors in previous interpretations of STEM/EELS experiments based on the assumption that the scattering cross-sections are the same as that of an isolated helium atom.

\section{Properties of helium atoms in bubbles and bulk condensed phases}

The ground $\left(1 s^{2}\right)$ to $1 s 2 p$ excitation energy in a bubble differs from that of 21.218 $\mathrm{eV}$ in a free helium atom being greater in both a bubble as well as in the bulk liquid or solid states. In previous work, both the excitation energy and the strength of the inelastic scattering due to that excitation have been measured with the object of deriving the helium density in a bubble [5, 7-10].

The excitation energy shifts $(\Delta E)$ can only be trusted to provide a reliable determination of a bubble density and hence pressure after the quantum mechanical predictions have been validated against experimental measurements for systems in which the bubble densities are known from entirely independent sources. Until recently only two such experimental data points were available: one for the bulk liquid [11] and the other for the "solid" [12]. In [13] excellent agreement with these results was found by using ab-initio electronic structure computations, each consisting of a self-consistent field ( $\mathrm{SCF}$ ) calculation augmented by dispersion and short range correlation contributions. The energy shift was explained by considering one of the helium atoms to be surrounded by a cluster of ground-state helium atoms. When the central helium atom is excited into the $1 s 2 p$ state, the $2 p$ orbital on the central atom overlaps the electron densities of the surrounding atoms. The Pauli principle requires that the wavefunction describing all the electrons in the system should be antisymmetrized. When the $2 p$ orbital is orthogonalized to the $1 s$ orbitals on the surrounding atoms, extra nodes are introduced into the $2 p$ orbital, which increases 


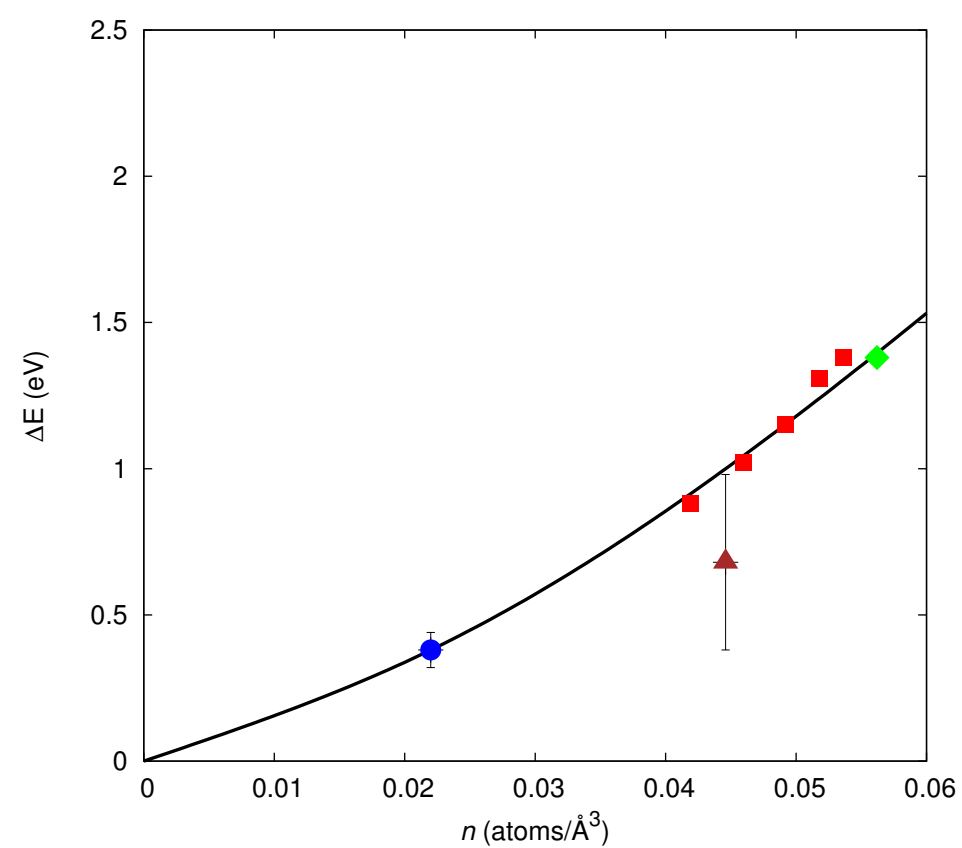

Figure 1.: Comparison of our ab-initio energy shift calculations using the "extended" model as described in [13] (solid line) with experimental data for the bulk liquid from [11] (circle) and [15] (squares) as well as for the bulk solid from [12] (triangle) and [14] (diamond).

its energy. The energy of the cluster can be reduced by using a more contracted $2 p$ orbital but the energy of the cluster is still greater than before the Pauli principle was taken into account.

In [13] the SCF wave functions were calculated in two models: a "basic" model in which the excited $2 p$ electron remains on the central helium atom with all neighboring atoms in the free-atom ground state, and an extended model in which the possibility of delocalization of the $2 p$ electron onto the surrounding atoms in the cluster was included. For the full details of the models used in our computations, the reader is referred to [13]. More extensive and accurate experimental data has been reported $[14,15]$ since the publication of [13]. We have repeated and confirmed the calculations in our earlier work and extended their range to allow for comparison with the more recent experimental results, and have found excellent agreement between theory and experiment, shown in Fig. 1.

At the time, 2001, of the publication of [13] there was only one experimental investigation which measured the properties of individual bubbles. The three quantities directly measured were the energy shift, the bubble diameter $d$, and the ratio $\frac{I_{p}}{I_{z}}$ where $I_{z}$ is the unscattered beam intensity and $I_{p}$ is the intensity in the He $1 \mathrm{~s} \rightarrow 2 \mathrm{p}$ peak. They presented values of the number density $n$ using the relation [16]

$$
n=\frac{I_{p}}{\sigma_{2 p} I_{z} d}
$$

by assuming a scattering cross section $\sigma_{2 p}$ that is derived from the free helium 
atom wavefunctions [17]. More recent measurements of $\Delta E$ for an extensive range of bubbles [7-9] differ very significantly from the bulk helium $\Delta E$ if the bubble densities are derived using the free atom sigma in (1). For example shifts of over 2 $\mathrm{eV}$ are reported [8] for bubbles with number densities derived using the free helium cross sections to be around 0.04 atoms $/ \AA^{3}$ whilst for this density in the bulk $\Delta E$ is only found to be of the order of $0.9 \mathrm{eV}$.

The results from our previous calculations showed that the mean squared radius of the $2 p$ orbital was significantly decreased with increasing density. Thus the free atom $\left\langle r^{2}\right\rangle$ of 31.56 a.u. is lowered to 15.05 a.u. for $n=0.05$ atoms $/ \AA^{3}$. These results indicate $\sigma_{2 p}$ might be significantly modified by its environment in both the bulk and the bubbles. In the first Born approximation, the cross section $\sigma_{2 p}$ for excitation of the target atom from the ground state to the $2 p$ excited state by a fast incident electron is given in $[17,18]$ by the equation

$$
\sigma_{2 p}=\frac{2 \pi}{E_{i} \Delta U_{n}} \int_{q_{\min }}^{q_{\max }} \frac{f_{2 p}(q)}{q} d q
$$

Here $E_{i}$ is the energy of the incident electron in the STEM experiment, $\Delta U_{n}$ is the energy required to excite a ground state helium atom to its $1 s 2 p{ }^{1} P$ state and $q_{\text {min }}$ and $q_{\max }$ are the miniumum and maximum values of the momentum transfer as given in $[1,17,18]$. In $(2) f_{2 p}(q)$ is the generalized oscillator strength (GOS)

$$
f_{2 p}(q)=2 \Delta U_{n} \frac{\left|\epsilon_{2 p}(q)\right|^{2}}{q^{2}}
$$

and $\epsilon_{2 p}(q)$ is the atomic form factor, given by the integral

$$
\epsilon_{2 p}(q)=\int \Psi_{2 p}^{*}\left(\mathbf{r}_{1}, \mathbf{r}_{2}\right) \sum_{j=1}^{2} e^{i \mathbf{q} \cdot \mathbf{r}_{j}} \Psi_{0}\left(\mathbf{r}_{1}, \mathbf{r}_{2}\right) d \mathbf{r}_{1} d \mathbf{r}_{2}
$$

Here $\Psi_{2 p}\left(\mathbf{r}_{1}, \mathbf{r}_{2}\right)$ and $\Psi_{0}\left(\mathbf{r}_{1}, \mathbf{r}_{2}\right)$ are the two-electron wavefunctions for the $1 s 2 p^{1} \mathrm{P}$ and $1 s^{2}$ states respectively. After expressing these two states in terms of single electron orbitals, (4) becomes

$$
\epsilon_{2 p}(q)=\sqrt{2}\left\langle\psi_{1 s}(\mathbf{r}) \mid \psi_{1 s 0}(\mathbf{r})\right\rangle\left\langle\psi_{2 p}(\mathbf{r})\left|e^{i \mathbf{q} \cdot \mathbf{r}}\right| \psi_{1 s 0}(\mathbf{r})\right\rangle,
$$

where $\psi_{1 s}(\mathbf{r})$ and $\psi_{1 s 0}(\mathbf{r})$ are the $1 s$ wavefunctions of the excited and ground states respectively. Although these two functions are not identical, their overlap $\left\langle\psi_{1 s}(\mathbf{r}) \mid \psi_{1 s 0}(\mathbf{r})\right\rangle$, which is explicitly evaluated in all our computations, is always very close to unity. This shows that the overwhelming majority of the environmental dependence of $\epsilon_{2 p}(q)$ arises from the compression of the $2 p$ orbital acting to increase its value closer to the nucleus where $\psi_{1 s 0}(\mathbf{r})$ is more significant. The term $\left\langle\psi_{2 p}(\mathbf{r})\left|e^{i \mathbf{q} \cdot \mathbf{r}}\right| \psi_{1 s 0}(\mathbf{r})\right\rangle$ in (5) is thereby enhanced.

We have calculated $\epsilon_{2 p}(q)$ for a range of densities from wavefunctions computed with the GAMESS-UK program [19] for helium atom clusters as described previously [13]. Fig. 2 shows how $\left|\epsilon_{2 p}(q)\right|$ is significantly enhanced on passing from the 
free atom through the $n$ value of 0.0286 atoms $/ \AA^{3}$ presented in [5] to a density of 0.05 atoms $/ \AA^{3}$. The accuracy of our free atom $\epsilon_{n}(q)$ computed from the Hartree Fock wave functions is shown by its very close agreement with the result derived from very accurate correlated wave functions [17].

The density dependence of the computed cross section is presented in Fig. 3. It is clear that the environmentally induced enhancements of the $\epsilon_{2 p}(q)$ are carried over into $\sigma_{2 p}$. It is notable that, even for moderate densities around 0.05 atoms $/ \AA^{3}$, the cross section is double that of the free helium atom.

Although the overwhelming majority of the variation in the calculated $\sigma_{2 p}$ values arises from the compression of the $2 p$ orbital, one other factor can in principle contribute to a density dependence of $\sigma_{2 p}$, namely the effect of $\Delta U_{n}$ on the integration limits $q_{\min }$ and $q_{\max }$ [5]. It should be noted that the $\Delta U_{n}$ in the dominator of (2) is cancelled after substitution of $(3)$ for $f_{2 p}(q)$. However in [5] a parametrized form for the free atom $f_{2 p}$ was used which implicitly contains a factor of $\frac{1}{\Delta U_{0}}$ (the free atom $\Delta U_{n}$ value) while the density dependent $\Delta U_{n}$ was used in (4). The $n$ dependence resulting from both these factors of $\sigma_{2 p}$ is seen from Fig. 3 to be very small. The $n$ dependence of this calculated $\sigma_{2 p}$ would be even smaller if the inconsistent treatment of the $\Delta U_{n}$ factors was removed.

The large density dependence of $\sigma_{2 p}$ presented in Fig. 3 clearly shows that any value of $n$ deduced from (1) using the free atom value for $\sigma_{2 p}$ as performed in $[5,7-10,20]$ cannot be trusted.

It is clear that

$$
\frac{I_{p}}{\sigma_{2 p} I_{z} d}
$$

cannot be taken to be an explicit function of $n$. It is, rather, an implicit function of the density since $\sigma_{2 p}$ is itself a function of density.

The quantities directly measured in the STEM experiments are $I_{p}, I_{z}$, and $d$. This shows that the purely experimental quantity related to (1) is the product $n \cdot \sigma_{2 p}$ given by

$$
\Sigma=\frac{I_{p}}{I_{z} d}
$$

\section{Comparison of theory and experiment via the $\Sigma$ values}

The quantities $\Sigma$ that were directly measured in STEM experiments can be reconstructed from the reported densities $n$ by multiplying the latter by the $\sigma_{2 p}$ value, derived from the free atom GOS, that was used to calculate $n$. However both $I_{p}$ and $I_{z}$ depend on the details of the experiments, namely on the collection angles determining the $q_{\min }$ and $q_{\max }$ entering (2) as well as on the energy and convergence angle of the incident beam. The latter parameter can be described in terms of an effective maximum collection angle using the CONCOR2 code of Egerton [1]. The relation (1) then shows, consitent with equation (2) for $\sigma_{2 p}$, that the value of the latter to be used to reconstruct the experimental $\Sigma$ depends on the collection angles. For each of the experimental data considered below, we derived the $\Sigma$ value from the free atom $\sigma_{2 p}$ computed using the collection angles which were 


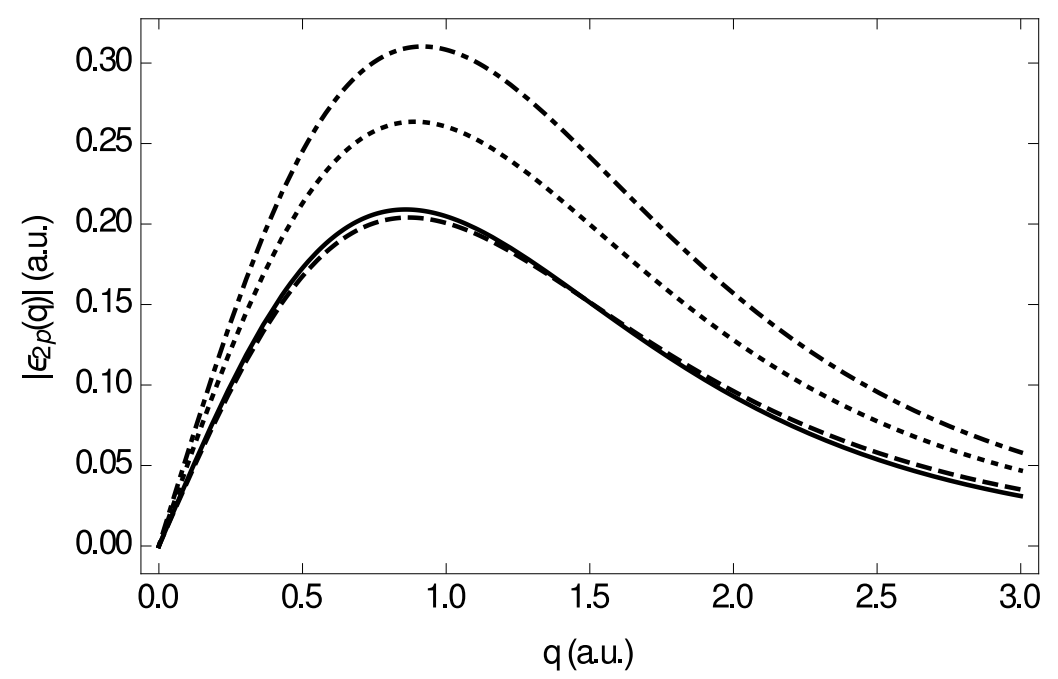

Figure 2.: A comparison of the atomic form factor for free helium (solid) with our calculation for free helium (dashed), our delocalized calculation for BCC helium at density $n=0.0286$ atoms $/ \AA^{3}$ (dotted), and our delocalized calculation for BCC helium at density $n=0.05$ atoms $/ \AA^{3}$ (dot-dashed). For free helium, the analysis in $[5]$ is entirely correct.

either reported in the literature or communicated to us from the authors of the original paper. $\Sigma$ values directly comparable to each of four sets of experiments were derived from the GOS generated by our computations by calculating the $\sigma_{2 p}$ (2) using the appropriately corresponding instrumental parameters. The differences in the latter necessitate presenting separately the four comparisons between theory and experiment (Figure 4).

The report [7] of the study of bubbles in a PdPt matrix presents two sets of data points which differ in that two densities are reported for the same $\Delta E$ measured at the centre of the same bubble. However the derivation of the densities involves some averaging over the scattering from different regions in the bubble. The first set of densities, called uncorrected, was derived using the method of [5] without modification. The second set of densities, labelled corrected, was produced because it was considered that the scattering properties of helium atoms in proximity to the metallic wall are modified. The $\Sigma$ values for corrected densities are plotted in Fig. 4a, the uncorrected values (not shown in Fig. 4a) would appear to the left of those shown, with this leftward move increasing from about $8 \%$ for the smallest $\Delta E$ to $16 \%$ for the largest. Thus application of the correction in [7] does not remove the large differences between the bubble and bulk $\Sigma / \Delta E$ relationships. It can be assumed that even though the application of this correction to the rest of the experimental data shown in Fig. 4 would lead to a smaller disagreement between theory and experiment, the disagreement would still be far too significant to be explained solely by this effect.

In the study [8] of bubbles in Martensitic steel, variations between the instrumental parameters in the different experiments produced a range of the $\sigma_{2 p}$ values used in (1) to deduce the densities. The limits of this range were $5.5 \times 10^{-4} \AA^{2}$ and $9.1 \times 10^{-4} \AA^{2}$. For both these $\sigma_{2 p}$ values we have calculated $\Sigma$ and plotted in 


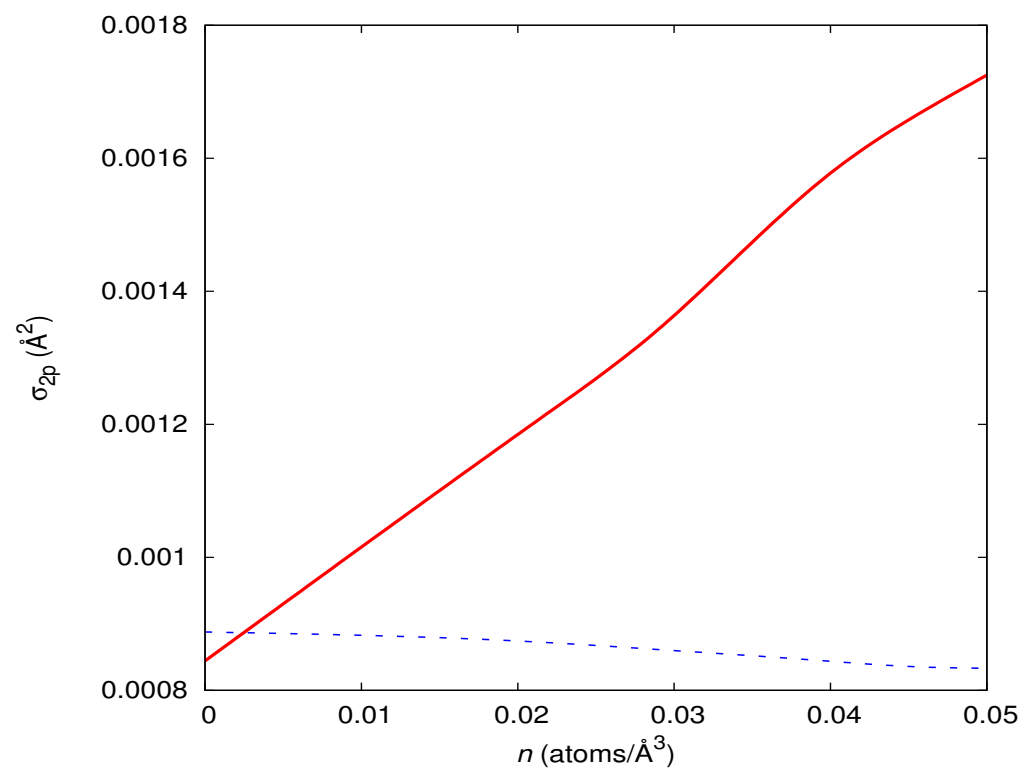

Figure 3.: Cross section plotted as a function of number density $n$ using the free atom wave function (dashed line) and the delocalized wave functions of the present work (solid line).

Fig. 4b their measured $\Delta E_{n}$ against $\Sigma$. The two theoretical curves shown in Fig. $4 \mathrm{~b}$ correspond to the two sets of experimental parameters which give rise to the reported limits of $\sigma_{2 p}$ in [8]. The differences between the theoretical and experimental $\Sigma$ presented in Figure $4 \mathrm{~b}$ are at least as large, if not larger, than those in Figure 4a.

The paper [5] of the study of bubbles in the PE16 alloy reports all the experimental details for the two bubbles studied. Our calculations showed that the effect of the differences in the collection angles of the two experiments is extremely small. Consequently the comparison in figure $4 \mathrm{c}$ between theory and experiment reports only the theoretical curve which is closer to the experiments. Again the differences between theory and experiment lie well outside the error bars reported for the latter.

For bubbles in a silicon matrix, theory is compared with the experiment [9] in Figure $4 \mathrm{~d}$. The clear differences between theory and experiment would only be reduced if a constant energy offset was included in the latter. The difficulty of accurately measuring small energy shifts has been discussed [10].

\section{Conclusions}

There are three main conclusions resulting from the present work. Firstly we have demonstrated that the values of the energy shifts predicted using the extended model of [13] are in excellent agreement with the laboratory measurements of the bulk. This encourages us to believe that the contractions of the $2 p$ orbital with increasing density have been correctly described and as a consequence to deduce that the the quantities directly measured in STEM experiments are implicitly rather than explicitly dependent on the density. Secondly we have shown that the contraction of the $2 p$ orbital causes $\sigma_{2 p}$ to increase with density very markedly.

Thirdly it has been shown that most existing STEM measurements for the bubbles 

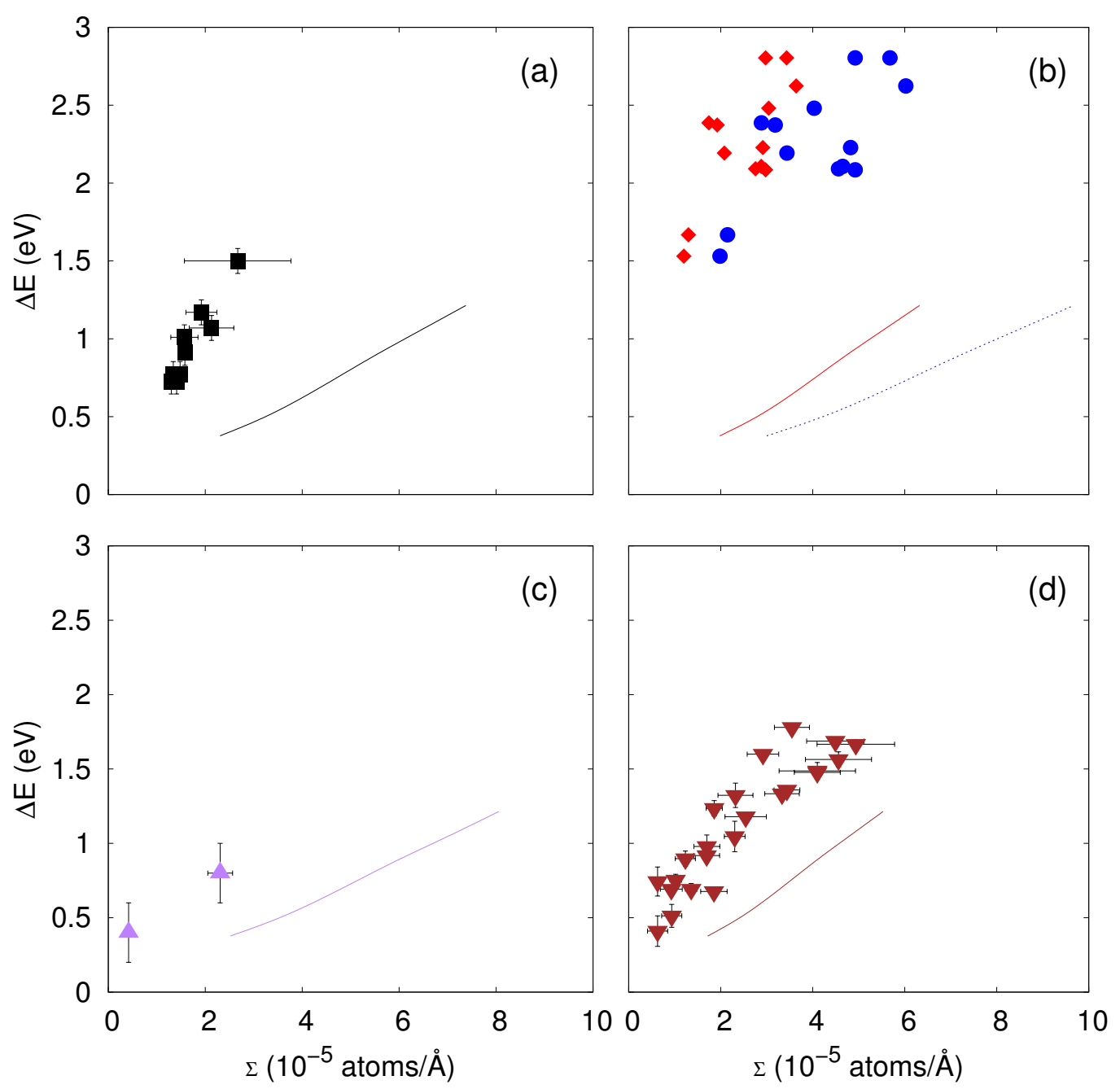

Figure 4.: Comparison of our theoretical values of $\Delta E$ as a function of $\Sigma$ (solid and dashed lines) with experimental data. Plotted in part (a) are the (corrected) experimental values of [7] (squares). In part (b) the upper (discs) and lower (diamonds) limiting values of [8] are both shown since only a range of values of $\sigma$ are given; the solid and dashed theoretical curves correspond to the diamonds and discs, respectively. In (c) the data from [5] is plotted (triangles), and in (d) the data from [9] (inverted triangles) is shown. The axes on all four plots shown are identical.

are inconsistent with our theoretical predictions, with the possible exception of the bubbles in silicon. This suggests either some systematic experimental problem with this type of measurement or that there may be physics present in the bubbles which is absent from the bulk. If the experimentally measured energy shifts in silicon needed to be corrected with a constant offset bringing theory and experiment into near agreement, it would follow that bubbles in silicon behaved differently from 
those in the three metals. This would provide further evidence for the influence on bubble properties from the encapsulating matrix.

Additionally, we note that there have been attempts to find a linear relationship between $\Delta E$ and $n[5,7-10]$, however this would not be consistent with our theory and indeed leads to a large error at higher densities.

\section{Acknowledgements}

TCN would like to thank Prof. Martyn Guest of Cardiff University for his invaluable assistance in getting the GAMESS-UK calculations to run smoothly and also Profs. Marie-Laure David and Laurent Pizzagalli of Université Poitiers and Prof. Mathieu Kociak of the Laboratory for Solid State Physics in Orsay for helpful correspondence regarding experimental details. NCP and TCN thank Alex Thom for his help in running some of the GAMESS calculations.

\section{Funding}

This research was supported by a grant from the Royal Society under the International Exchange Scheme. TCN gratefully acknowledges funding from EPSRC under grant number EP/LO15722/1.

\section{References}

[1] R.F. Egerton, Electron Energy Loss Spectroscopy in the Electron Microscope, Plenum, New York, 1996.

[2] P. Rez, Cross-sections for energy loss spectrometry, Ultramicroscopy 9 (1982) p. 283.

[3] J.P. Connerade, Structure and Properties of Atomic Clusters, p. 53.

[4] H. Trinkaus and B.N. Singh, Helium accumulation in metals during irradiation-where do we stand?, J. Nucl. Mater. 323 (2003) p. 229.

[5] C.A. Walsh, J. Yuan and L.M. Brown, A procedure for measuring the helium density and pressure in nanometre-sized bubbles in irradiated materials using electron-energyloss spectroscopy, Phil. Mag. A 80 (2000) p. 1507.

[6] H. Trinkaus, Energetics and formation kinetics of helium bubbles in metals, Radiation Effects 78 (1983) p. 189.

[7] D. Taverna, M. Kociak, O. Stéphan, A. Fabre, E. Finot, B. Décamps and C. Colliex, Probing Physical Properties of Confined Fluids within Individual Nanobubbles, Phys. Rev. Lett. 100 (2008).

[8] S. Fréchard, M. Walls, M. Kociak, J.P. Chevalier, J. Henry and D. Gorse, Study by EELS of helium bubbles in a martensitic steel, J. Nucl. Mater. 393 (2009) p. 102.

[9] M.L. David, F. Pailloux, V. Mauchamp and L. Pizzagalli, In situ probing of helium desorption from individual nanobubbles under electron irradiation, Appl. Phys. Lett. 98 (2011) p. 171903.

[10] K. Alix, M.L. David, G. Lucas, D.T.L. Alexander, F. Pailloux and L. Pizzagalli, Gentle quantitative measurement of helium density in nanobubbles in silicon by spectrum imaging, Micron 77 (2015) p. 57.

[11] C.M. Surko, G.J. Dick, F. Reif and W.C. Walker, Spectroscopic Study of Liquid Helium in the Vacuum Ultraviolet, Phys. Rev. Lett. 23 (1969) p. 842.

[12] N. Schell, R.O. Simmons, A. Kaprolat, W. Schulke and E. Burkel, Electronic Exci- 
tations in hcp ${ }^{4} \mathrm{He}$ at $61.5 \mathrm{MPa}$ and $4.3 \mathrm{~K}$ Studied by Inelastic X-Ray Scattering Spectroscopy, Phys. Rev. Lett. 74 (1995) p. 2535.

[13] N.C. Pyper, D.W. Essex and C.T. Whelan, The $1 s^{2} \rightarrow 1 s 2 p\left({ }^{1} P\right)$ excitation energy for helium atoms in liquid helium, solid helium and helium bubbles in metals, Phil. Mag. B 81 (2001) p. 91.

[14] D.A. Arms, R.O. Simmons, M. Schwöerer-Bohning, A.T. Macrander and T.J. Graber, Exciton Dispersion and Electronic Excitations in hcp ${ }^{4} \mathrm{He}$, Phys. Rev. Lett. 87 (2001) p. 156402 .

[15] D.A. Arms, T.J. Graber, A.T. Macrander, R.O. Simmons, M. Schwöerer-Bohning and Y. Zhong, Excitons in bulk liquid ${ }^{4}$ He, Phys. Rev. B 71 (2005) p. 233107.

[16] A.J. McGibbon and L.M. Brown, Microanalysis of nanometer-sized helium bubbles using parallel-detection EELS in a STEM, Trans. R. Microsc. Soc. 1 (1990) p. 23.

[17] M. Inokuti, Inelastic Collisions of Fast Charged Particles with Atoms and Mocules-The Bethe Theory Revisited, Rev. Mod. Phys 43 (1971) p. 297.

[18] A. Burgess and J.A. Tully, On the Bethe approximation, J. Phys. B 11 (1978) p. 4271.

[19] GAMESS-UK is a package of ab initio programs. See: "http://www.cfs.dl.ac.uk/gamess-uk/index.shtml", M. F. Guest, I. J. Bush, H. J. J. van Dam, P. Sherwood, J. M. H. Thomas, J. H. van Lenthe, R. W. A. Havenith, J. Kendrick, "The GAMESS-UK electronic structure package: algorithms, developments and applications", Molecular Physics, Vol. 103, No. 6-8, 20 March-20 April 2005, 719-747 .

[20] R. Schierholz, B. Lacroix, V. Godinho, J. Caballero-Hernández, M. Duchamp and A. Fernández, STEM-EELS analysis reveals stable high-density He in nanopores of amorphous silicon coatings deposited by magnetron sputtering, Nanotechnology 26 (2015) pp. 1-10.

[21] A.A. Lucas, J.P. Vigneron, S.E. Donnelly and J.C. Rife, Theoretical interpretation of the vacuum ultraviolet reflectance of liquid helium and of the absorption spectra of helium microbubbles in aluminum, Phys. Rev. B 28 (1983) p. 2485. 